\title{
BLAST05: POWER SPECTRA OF BRIGHT GALACTIC CIRRUS AT SUBMILLIMETER WAVELENGTHS
}

\author{
Arabindo Roy ${ }^{1}$, Peter A. R. Ade ${ }^{2}$, James J. Bock ${ }^{3,4}$, Edward L. Chapin ${ }^{5}$, Mark J. Devlin ${ }^{6}$, Simon R. Dicker 6 , \\ Matthew Griffin ${ }^{2}$, Joshua O. Gundersen ${ }^{7}$, Mark Halpern ${ }^{5}$, Peter C. Hargrave ${ }^{2}$, David H. Hughes ${ }^{8}$, Jeff Klein ${ }^{6}$, \\ Gaelen Marsden $^{5}$, Peter G. Martin ${ }^{1,9}$, Philip Mauskopf $^{2}$, Marc-Antoine Miville-Deschênes ${ }^{10}$, CAlvin $^{2}$ \\ B. Netterfield ${ }^{1,11}$, Luca Olmi ${ }^{12,13}$, Guillaume Patanchon ${ }^{14}$, Marie Rex ${ }^{6}$, Douglas Scott ${ }^{5}$, Christopher Semisch ${ }^{6}$, \\ Matthew D. P. Truch ${ }^{15}$, Carole Tucker ${ }^{2}$, Gregory S. Tucker ${ }^{15}$, Marco P. Viero $^{1}$, and Donald V. Wiebe ${ }^{5}$ \\ ${ }^{1}$ Department of Astronomy \& Astrophysics, University of Toronto, 50 St. George Street, Toronto, ON M5S 3H4, Canada; aroy@ cita.utoronto.ca \\ ${ }^{2}$ Department of Physics \& Astronomy, Cardiff University, 5 The Parade, Cardiff, CF24 3AA, UK \\ 3 Jet Propulsion Laboratory, Pasadena, CA 91109-8099, USA \\ ${ }^{4}$ Observational Cosmology, MS 59-33, California Institute of Technology, Pasadena, CA 91125, USA \\ ${ }^{5}$ Department of Physics \& Astronomy, University of British Columbia, 6224 Agricultural Road, Vancouver, BC V6T 1Z1, Canada \\ ${ }^{6}$ Department of Physics and Astronomy, University of Pennsylvania, 209 South 33rd Street, Philadelphia, PA 19104, USA \\ ${ }^{7}$ Department of Physics, University of Miami, 1320 Campo Sano Drive, Carol Gables, FL 33146, USA \\ ${ }^{8}$ Instituto Nacional de Astrofísica Óptica y Electrónica (INAOE), Aptdo. Postal 51 y 72000 Puebla, Mexico \\ ${ }^{9}$ Canadian Institute for Theoretical Astrophysics, University of Toronto, 60 St. George Street, Toronto, ON M5S 3H8, Canada \\ ${ }^{10}$ Institut d' Astrophysique Spatiale, UMR8617, Université Paris-Sud, F-91405, Orsay, France \\ ${ }^{11}$ Department of Physics, University of Toronto, 60 St. George Street, Toronto, ON M5S 1A7, Canada \\ ${ }^{12}$ Physics Department, University of Puerto Rico, Rio Piedras Campus, P.O. Box 23343, UPR station, San Juan, Puerto Rico \\ ${ }^{13}$ Istituto di Radioastronomia, Largo E. Fermi 5, I-50125, Firenze, Italy \\ ${ }^{14}$ Laboratoire APC, 10, rue Alice Domon et Léonie Duquet 75205 Paris, France \\ 15 Department of Physics, Brown University, 182 Hope Street, Providence, RI 02912, USA \\ Received 2009 October 5; accepted 2009 November 19; published 2009 December 23
}

\begin{abstract}
We report multi-wavelength power spectra of diffuse Galactic dust emission from Balloon-borne Large Aperture Submillimeter Telescope observations at 250, 350, and $500 \mu \mathrm{m}$ in Galactic plane fields in Cygnus X and Aquila. These submillimeter power spectra statistically quantify the self-similar structure observable over a broad range of scales and can be used to assess the cirrus noise which limits the detection of faint point sources. The advent of submillimeter surveys with the Herschel Space Observatory makes the wavelength dependence a matter of interest. We show that the observed relative amplitudes of the power spectra can be related through a spectral energy distribution (SED). Fitting a simple modified black body to this SED, we find the dust temperature in Cygnus $\mathrm{X}$ to be $19.8 \pm 1.5 \mathrm{~K}$ and in the Aquila region $16.8 \pm 0.8 \mathrm{~K}$. Our empirical estimates provide important new insight into the substantial cirrus noise that will be encountered in forthcoming observations.
\end{abstract}

Key words: balloons - ISM: clouds - ISM: structure - submillimeter

Online-only material: color figures

\section{INTRODUCTION}

Energy is being injected continually into the interstellar medium (ISM) through spiral shocks, violent outflows from massive protostars, stellar winds, expanding $\mathrm{H}$ II regions, and supernova explosions. The result is a turbulent medium where the dust is well mixed in the structures produced. The emission from the relatively nearby ISM at high Galactic latitude is known as Galactic Cirrus. Cirrus-like structure in the brighter emission near the Galactic plane has been called "interstellar froth" (Waller \& Boulanger 1994); this might be an interesting distinction with a physical basis, but here we will simply use the term "cirrus". The dynamics of the ISM appear to have made the distribution of density structures self-similar, with fluctuations in column density and surface brightness present on all observable scales, though decreasing toward smaller scales. Statistical description of random fluctuations through structure functions is an important method of extracting physical properties hidden in diffuse emission as well as for providing a quantitative measure to compare with simulations. A common statistical tool used to estimate the level of cirrus noise is the power spectrum. This is valid for Gaussian random fields. However, Gautier et al. (1992) found evidence for non-Gaussianity and recent studies by Miville-Deschênes et al. (2007) have revealed non-vanishing skewness and excess kurtosis in the underlying brightness fluctuation fields. Nevertheless, for estimating the variance the power spectrum is still indicative.

Using power spectra, Gautier et al. (1992) quantified how fluctuations associated with Galactic cirrus are a source of confusion noise which limits the detectability of point sources. Even with the improved angular resolution of imagers like SPIRE and PACS on the Herschel Space Observatory ${ }^{16}$ (hereafter Herschel), this "cirrus noise" remains important, often dominant. Therefore, to assess the noise it is vital to know the statistical properties of the interstellar diffuse emission at the relevant wavelengths.

A number of other statistical analyses of emission have been carried out on the basis of power spectra for high-latitude clouds, e.g., by Kiss et al. (2003), Jeong et al. (2005), and Miville-Deschênes et al. (2007). In this paper, we analyze diffuse dust emission in the Galactic plane and present for the first time multi-wavelength power spectra in the submillimeter, based on observations in Cygnus X (Cyg X) and Aquila with the Balloon-borne Large Aperture Submillimeter Telescope (BLAST; Pascale et al. 2008) at 250, 350, and $500 \mu \mathrm{m}$. We

\footnotetext{
16 http://www.esa.int/SPECIALS/Herschel/index.html
} 
also analyze the same regions at 100 and $60 \mu \mathrm{m}$ using infrared interferometer spectrometer (IRIS), IRAS data reprocessed by Miville-Deschênes \& Lagache (2005). Compared to highlatitude studies, analyzing diffuse emission in the Galactic plane in terms of the structure of the ISM resulting from its turbulent properties is more challenging because of the long path lengths, high column density, high star formation rate, and contamination by compact sources. In addition, in the Galactic plane, self-gravity can play an important role in shaping diffuse structures at smaller scales. Of particular importance are the potentially strong spatial variations of the radiation field due to star formation activity. As discussed further in Section 6.1, this could produce variations in the dust emission independently of any changes in structure of the ISM.

Our paper is organized as follows. We begin with a brief description of BLAST observations (Section 2) and then introduce important aspects of the power spectrum and accompanying cirrus noise (Section 3). We analyze IRIS data in Section 4, placing this in the context of earlier studies and providing a short-wavelength reference for our submillimeter studies. In Section 5, we examine the BLAST data: the noise; effect of the point-spread function (PSF) or beam; removal of compact sources; and the exponents and amplitudes of the submillimeter power spectra. We estimate the cirrus noise for these maps and compare it with the completeness depth of the BLAST05 pointsource catalogs at $250 \mu \mathrm{m}$. In Section 5.6, we also discuss some implications for related approved observations with Herschel. We show in Section 6 how the observed wavelength dependence of the amplitude of the power spectrum can be understood as a straightforward consequence of the spectral energy distribution (SED) of the dust, and we fit a simple modified black body to estimate the dust temperature. Our empirical results provide new insight into what cirrus noise might be expected in submillimeter observations.

\section{OBSERVATIONS}

In 2005, the BLAST05 (Pascale et al. 2008)) made unbiased surveys in targeted regions of the Galactic plane. Chapin et al. (2008) analyzed a $4 \mathrm{deg}^{2}$ field in Vulpecula, and here we used data from the two largest surveys. Aquila (A. Rivera-Ingraham et al. 2010, in preparation) is a $6 \mathrm{deg}^{2}$ region observed for $6.1 \mathrm{hr}$, while Cyg X (A. Roy et al. 2010, in preparation) covers $10 \mathrm{deg}^{2}$ observed over $10.6 \mathrm{hr}$. Both have good cross-linking from orthogonal scanning. The maps were made with the SANEPIC algorithm (Patanchon et al. 2008) and were calibrated using the procedure discussed in Truch et al. (2008).

Although a $2 \mathrm{~m}$ telescope, BLAST05 produced maps of only $3^{\prime}$ resolution (see Section 5.2) due to an anomalous $\mathrm{PSF}$, corrupted by some uncharacterized combination of mirror distortion and de-focus (Truch et al. 2008). ${ }^{17}$ Nevertheless, maps from the 2005 flight have high signal to noise and are oversampled with $15^{\prime \prime}$ pixels, so that Lucy-Richardson (L-R) deconvolution can be used to improve the resolution significantly (A. Roy et al. 2010, in preparation). This goal is particularly important for extracting point sources (Section 5.3) but otherwise not essential for the study of diffuse emission. We analyzed the two largest surveys, selecting in Aquila a square sub-field of size 1.83 centered on $l=45.85, b=-0.12$ and a similar-sized field in Cyg X centred on $l=79.89, b=0.47$.

\footnotetext{
17 This problem was fixed for the 2006 flight from Antartica (Truch et al. 2009).
}

The latter does not include the brighter star-forming regions to the east containing W75-N and DR21 (Schneider et al. 2006).

We also analyzed IRIS maps at 100 and $60 \mu \mathrm{m}$ toward these selected regions. These maps have $\sim 4^{\prime}$ resolution on 1.5 pixels (Miville-Deschênes et al. 2002) and we used the version in which the sources have been removed by the technique described by Miville-Deschênes \& Lagache (2005).

\section{POWER SPECTRUM AND CIRRUS NOISE}

The power spectrum is the Fourier transform of the autocorrelation function of the intensity map $I(x, y)$. In $k$-space, $P\left(k_{x}, k_{y}\right)$ is simply related to an image by

$$
P\left(k_{x}, k_{y}\right)=\left\langle\tilde{I}\left(k_{x}, k_{y}\right) \tilde{I}^{\star}\left(k_{x}, k_{y}\right)\right\rangle,
$$

where $\tilde{I}\left(k_{x}, k_{y}\right)$ is the Fourier transform of the image and $\tilde{I}^{\star}$ its complex conjugate. We used the IDL routine fast Fourier transform (FFT) to compute the two-dimensional Fourier transforms. To ensure a smooth periodic boundary condition near the edges (Miville-Deschênes et al. 2002), the maps were apodized by a sine function over a range $10 \%$ the width of the map. The power spectrum $P(k)$ is obtained by averaging $P\left(k_{x}, k_{y}\right)$ over an annulus placed at $k=\sqrt{k_{x}^{2}+k_{y}^{2}}$.

In practice, contributions to the total power spectrum come not only from diffuse dust emission, but also point sources, the cosmic infrared background (CIB), and the noise. When these components are statistically uncorrelated, the total power spectrum can be expressed as (Miville-Deschênes et al. 2007)

$$
P(k)=\Gamma(k)\left[P_{\text {cirrus }}(k)+P_{\text {source }}(k)+P_{\mathrm{CIB}}(k)\right]+N(k),
$$

where $\Gamma(k)$ is the power spectrum of the PSF (the square of the modulus of the two-dimensional Fourier transform of the PSF), which decays at large $k$. For the bright Galactic plane fields targeted here, the contribution from the CIB to the power spectrum is negligible. While the noise is measurable (Section 5.5), it too makes an insignificant contribution.

Gautier et al. (1992), followed by many other authors (Kiss et al. 2001, 2003; Miville-Deschênes et al. 2002), have shown that the power spectrum of Galactic cirrus follows a power law,

$$
P(k)=P\left(k_{0}\right)\left(k / k_{0}\right)^{\alpha},
$$

quantified by an amplitude $P_{0} \equiv P\left(k_{0}\right)$ at some fiducial $k_{0}$ and an exponent $\alpha$ that is typically -3 (Miville-Deschênes et al. 2007). From their analysis, the associated "cirrus noise" for a telescope with mirror diameter $D$ working at wavelength $\lambda$ can be quantified as

$$
\begin{aligned}
\sigma_{\text {cirrus }} & =100(r / 1.6)^{2.5}\left(\frac{\lambda / 250 \mu \mathrm{m}}{D / 3.5 \mathrm{~m}}\right)^{2.5} \\
{[P(k} & \left.\left.=0.1 \operatorname{arcmin}^{-1}\right) / 10^{-3} \mathrm{MJy}^{2} \mathrm{sr}^{-1}\right]^{0.5} \mathrm{mJy} .
\end{aligned}
$$

Here our assumptions about the measurement strategy for point sources are the same as made by Helou \& Beichman (1990) and adopted by Kiss et al. (2001), which in the notation of Gautier et al. (1992) are a "resolution ratio" $r=d /(\lambda / D)$ of 1.6 , where $d$ is the measuring aperture, and a reference annulus with "separation ratio" 2 . Also $\alpha=-3$, close to what we find below. The beam-related factor quantifies the effect of a smaller beam probing smaller spatial scales where the power in the fluctuations is weaker. 
If the sources being measured are extended, as will be the case in many Galactic surveys, then the measuring aperture needs to be larger. The main consequence of this on increasing $\sigma_{\text {cirrus }}$ is captured by the factor $r^{2.5}$ for the range of interest (see also Figure 3 in Gautier et al. 1992).

Working with $100 \mu \mathrm{m}$ IRAS data on fields of different average surface brightness $\left\langle I_{100}\right\rangle$, and adopting a fiducial scale $k_{0}=0.01 \operatorname{arcmin}^{-1}$ at which the amplitude is $P_{100}$, Gautier et al. (1992) found the trend that $P_{100}=C\left\langle I_{100}\right\rangle^{3}$, where $C$ is a proportionality constant. If this is substituted in the above, we recover the formula given by Helou \& Beichman (1990) and evaluated by Kiss et al. (2001) estimating the cirrus noise for different levels of cirrus brightness. Note that the cirrus noise estimate described by Miville-Deschênes et al. (2007) used a slightly different definition of the noise, as well as incorporating a trend giving a slightly steeper power law with increasing $\left\langle I_{100}\right\rangle$, and in effect a lower $C$, which together conspire to lower the noise estimate by about a factor of 6 for bright cirrus.

For reasons discussed below, both estimating $P_{100}$ from $\left\langle I_{100}\right\rangle$ and scaling it to the appropriate wavelength of observation are problematical. Therefore, if possible the exponent and amplitude of $P(k)$ should be measured directly for the field of interest at the relevant wavelength. Measuring and normalizing at a scale as close as possible to the beam also avoid issues of extrapolation. With Herschel in mind, we have chosen $k=0.1 \operatorname{arcmin}^{-1}$. The normalization of the amplitude in Equation (4) anticipates what we find at $250 \mu \mathrm{m}$ for the two bright fields we examined.

\section{IRIS POWER SPECTRA}

Our primary goals are to analyze statistical fluctuations of diffuse dust emission in the Galactic plane using the power spectrum, and to measure the wavelength dependence of the amplitude of this power spectrum. To connect with previous work and provide a reference at shorter wavelengths, we begin with IRIS. IRIS (like IRAS) data come in "plates" 12.5 on a side. We computed the power spectrum for the entire plate containing the Aquila field (plate 263) but because of a gap in the IRAS sky coverage near Cyg X (plate 361) we had to settle for a smaller region, $6^{\circ}$ on a side in Galactic coordinates centred on $l=80.69$ and $b=0.76$. Binned estimates of $P(k)$ and their uncertainties were evaluated as in Section 5.4.

The power spectrum $P(k)$ for the Aquila field at $100 \mu \mathrm{m}$ is shown in Figure 1. The behavior of $P(k)$ is very much like that seen at higher latitudes and with BLAST (Section 5.5). To find $P_{\text {cirrus }}(k)$ from Equation (2), we need an estimate of the power spectrum of the noise, $N(k)$. This can be achieved using maps of the differences of independent IRAS maps (HCONs; MivilleDeschênes et al. 2002) after source removal (Miville-Deschênes $\&$ Lagache 2005) and is shown in the lower curve in Figure 1. There is a feature at $k \sim 0.08 \operatorname{arcmin}^{-1}$ which is not seen in $P(k)$. This arises because of striping in the HCONs which affects the difference maps but is smoothed out in the average of the HCONs. To eliminate this spurious feature in the noise power spectrum to be subtracted, we have simply fit a power law for the range $k<0.06 \mathrm{arcmin}^{-1}$ and extrapolated this to higher $k$ (see the lower dashed curve). The exponent is $-1.5 \pm 0.2$; Miville-Deschênes \& Lagache (2005) found a range from -1.5 to 0 . The extrapolation meets $P(k)$ at the highest $k$ showing that it is a reasonable estimate of the noise, including that induced by the source removal technique which is greatest in Galactic plane fields like this where the surface density of sources is large. For the beam $\Gamma(k)$ we used a Gaussian as specified by Miville-Deschênes \& Lagache (2005) which is clearly just an

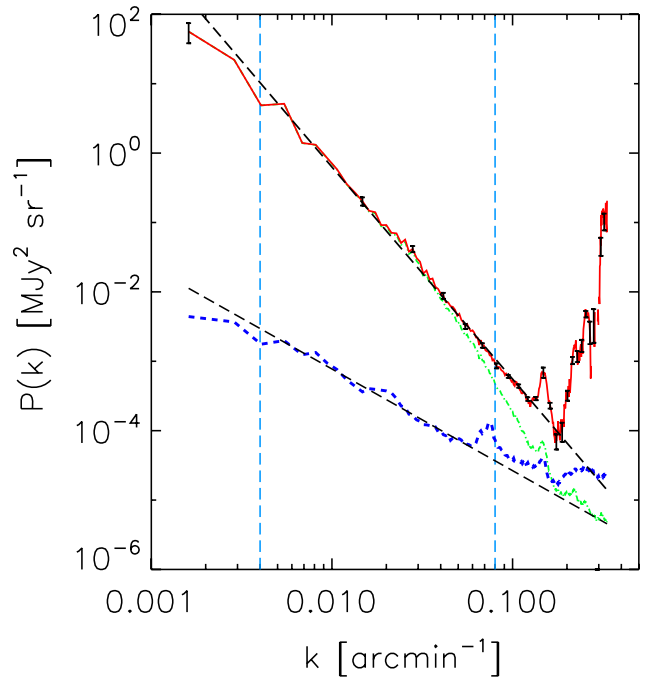

Figure 1. IRIS $100 \mu \mathrm{m}$ power spectrum (dot-dash curve) of a square 12.5 on a side in Aquila spanning the Galactic plane. $P_{\text {cirrus }}$ (solid) is obtained by subtracting $N(k)$ and dividing by $\Gamma(k)$. The estimate of $N(k)$ adopted (lower dashed power law) was obtained using differences of independent IRIS maps (HCONs; see the text). The error bars, plotted only every tenth point for clarity, do not account for the imperfect approximation to the beam whose effect is clearly present at large $k$. We fit the power law (dashed line) over the range $0.004 \operatorname{arcmin}^{-1}<\mathrm{k}<0.08 \mathrm{arcmin}^{-1}$ marked by the vertical dashed lines. The exponent is $-3.06 \pm 0.02$ and $P_{100}=0.62 \pm 0.02 \mathrm{MJy}^{2} \mathrm{sr}^{-1}$.

(A color version of this figure is available in the online journal.)

approximation to the complicated effective beam of the images formed from the IRAS time streams. In any case, neither the noise estimate nor the fine details of the beam are critical, because here we use only the part of the power spectrum at lower $k<0.08 \operatorname{arcmin}^{-1}$. We do not compensate for the apodization of the image edges, but instead simply avoid small values of $k<0.004 \mathrm{arcmin}^{-1}$ where the power is significantly affected (Miville-Deschênes et al. 2002). Figure 1 confirms empirically that this is a good choice for the range of $k$ used for the powerlaw fit. The nonlinear weighted fits were carried out with the IDL routine MPFIT (Markwardt 2009).

Figure 2 shows the power spectrum for the square $6^{\circ}$ region in Cyg X. Here the IRAS map coverage in the individual HCONs is not complete, so it is not possible to make a direct estimate of $N(k)$. However, comparison of the rms of the difference maps (in the areas covered) indicates that at the highest $k, N(k)$ should have a level comparable to $P(k)$, as found in Aquila. Therefore, an approximation to $N(k)$ is to extrapolate this to lower $k$ along a power law, as show in the figure. Again, this is not critical to $P_{\text {cirrus }}(k)$ given the conservative range used for the power-law fit.

For $100 \mu \mathrm{m}$ we find an exponent $\alpha$ of $-2.67 \pm 0.09$ in Cyg $X$ and $-3.06 \pm 0.02$ in Aquila, where the errors are statistical based on the fit to the data. The systematic errors, from the estimate adopted for $N(k)$ and from the (conservative) choice of upper limit in the range of $k$ used for the fit, are comparable. Miville-Deschênes et al. (2007) found that the steepness of the power spectrum increases as a slow function of $\left\langle I_{100}\right\rangle$. For the two fields $\left\langle I_{100}\right\rangle$ is 260 and $120 \mathrm{MJy} \mathrm{sr}^{-1}$, respectively, and the expected exponents from this trend are -3.4 and -3.3 , slightly steeper than what we find. Note however that they find a scatter of 0.3 about their trend.

We find $P_{100}=1.5 \pm 0.2$ and $0.62 \pm 0.02 \mathrm{MJy}^{2} \mathrm{sr}^{-1}$ for $\mathrm{Cyg}$ $\mathrm{X}$ and Aquila, respectively. These can be compared to values of 4.3 and $0.59 \mathrm{MJy}^{2} \mathrm{sr}^{-1}$ from the trend in Miville-Deschênes 


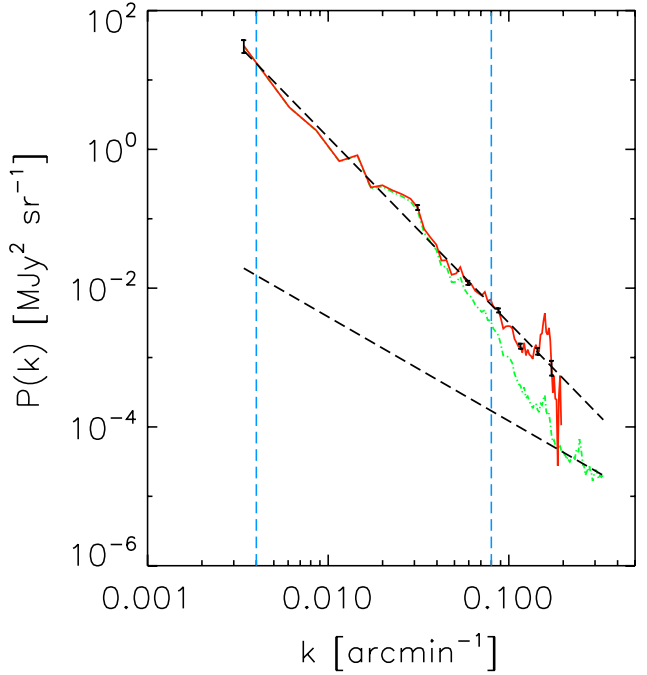

Figure 2. Same as Figure 1 but for a smaller square $6^{\circ}$ region in Cyg X. The exponent is $-2.67 \pm 0.09$ and $P_{100}=1.5 \pm 0.2 \mathrm{MJy}^{2} \mathrm{sr}^{-1}$.

(A color version of this figure is available in the online journal.)

et al. (2007), about which there is a factor of 3 scatter at high $\left\langle I_{100}\right\rangle$. Again the agreement is better for the Aquila field, despite the fact that there are significant asymmetries in the image (the bright swath of the Galactic plane and residual striping) which make the two-dimensional power spectrum not quite circularly symmetric.

For more direct comparison with the BLAST power spectra, we computed the IRIS $P_{\text {cirrus }}(k)$ for the smaller BLAST subfields. The exponents of the power spectra are $-3.14 \pm 0.27$ and $-2.92 \pm 0.17$, respectively. These are harder to measure, given the smaller dynamic range, but do not appear to have changed significantly despite the larger $\left\langle I_{100}\right\rangle$ values (430 and $330 \mathrm{MJy} \mathrm{sr}^{-1}$ ). Expressing amplitudes as $P_{100}$ (though $k=0.01$ $\operatorname{arcmin}^{-1}$ is beyond our range in these small sub-fields), we find $3.1 \pm 1.3$ and $0.75 \pm 0.11 \mathrm{MJy}^{2} \mathrm{sr}^{-1}$, respectively, compared to trend values of 29 and $13 \mathrm{MJy}^{2} \mathrm{sr}^{-1}$. Because of the $P^{1 / 2}$ dependence in Equation (4), predictions of cirrus noise depend less strongly on any deviations from the trends. Nevertheless, these results illustrate the important point that when attempting to assess the cirrus noise, one should, if possible, measure the exponent and amplitude near the spatial frequencies of interest.

Because the cirrus noise is wavelength dependent, ideally $P_{0}$ would be measured at each relevant wavelength too. The amplitude might in principle be scaled, say from $P_{100}$. Not all scaling prescriptions in the literature can be valid, however, and we discuss our recommendation in Section 6.

\section{BLAST POWER SPECTRA}

\subsection{Noise Power Spectrum}

In the BLAST map-making procedure (Patanchon et al. 2008), a variance map $V(x, y)$ is produced based on the noise in the time-stream data and the map coverage by the bolometers in the arrays. For these BLAST surveys, the resulting maps of $V(x, y)$ are quite uniform. A realization of the noise map $N(x, y)$ can be constructed for each independent, uncorrelated pixel from $N(x, y)=\sqrt{V(x, y)} v$, where $v$ is a Gaussian random variable with unit standard deviation and zero mean. Given the relative uniformity of the variance map, the resulting simulated noise map is close to white on all scales and thus the power spectrum of this noise is quite flat (see the example for Cyg X

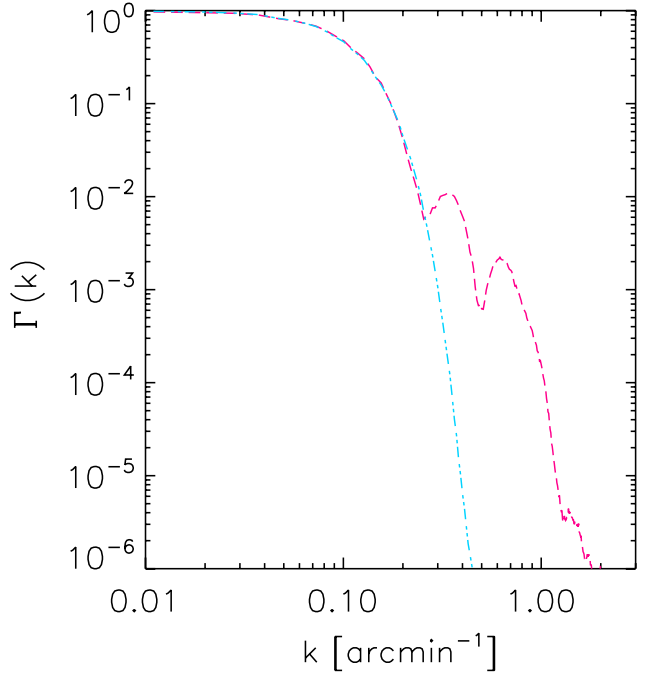

Figure 3. $\Gamma(k)$ for BLAST05 in Cyg $\mathrm{X}$ at $250 \mu \mathrm{m}$ (dashed curve). The dot-dash line shows how this can be approximated by the power spectrum of a Gaussian PSF of FWHM 3!3.

(A color version of this figure is available in the online journal.)

at $250 \mu \mathrm{m}$ in Figure 6). At large $k$, the total power spectrum decays to this noise level, because of the combined effects of the decreasing power in the diffuse cirrus emission and the PSF. There is also a component of low-frequency noise in the map arising from the very long timescale $1 / f$ noise present in the time streams. However, in cross-linked maps produced using data from multiple scanning directions this is greatly reduced by the SANEPIC algorithm (e.g., Figure 10 in Patanchon et al. 2008 ) and is not important here because the cirrus signal at small $k$ is so large.

\subsection{Effect of the Beam}

The BLAST05 PSF in telescope coordinates is shown in Truch et al. (2008). A synthetic beam can be made for a particular map, taking into account the scan angles and coverage (Chapin et al. 2008). Because this synthetic beam is not known out to the full size of the map, we have not derived its power spectrum $\Gamma(k)$ directly. Instead, we convolved the noise map with the PSF directly, found the power spectrum of that map, and divided by the power spectrum of the noise map. Figure 3 shows $\Gamma(k)$ for the BLAST05 PSF at $250 \mu \mathrm{m}$ for the Cyg X field. This has a number of features characterizing scales seen in the corrupted PSF. Clearly, the observed $P(k)$ will be seriously suppressed at high $k$ and so we will use data only for $k<0.2$ $\operatorname{arcmin}^{-1}$. At these scales, where the beam correction is not too large and so both more reliable and of less import, $\Gamma(k)$ can be described by a Gaussian with $\sigma_{\Gamma}=0.08 \operatorname{arcmin}^{-1}$. Using the Fourier relation $2 \sqrt{2} \pi \sigma_{\Gamma} \sigma_{\mathrm{b}}=1$, this corresponds to a Gaussian beam of $\sigma_{\mathrm{b}}=1.4$, or FWHM $=3.3$, slightly smaller than for IRIS. This is close to the 3.1 full width at half-power found by Truch et al. (2008). The corruption of the BLAST05 PSF is such that the initial falloff in $\Gamma(k)$ is rather similar for the three wavelengths, and for both fields.

\subsection{Source Removal}

In the Galactic plane, the power spectrum is seriously contaminated by compact sources. To remove them, we first deconvolve the BLAST maps using the L-R method which, importantly, conserves flux. Compact sources are of size $\sim 1.5$ in these maps 


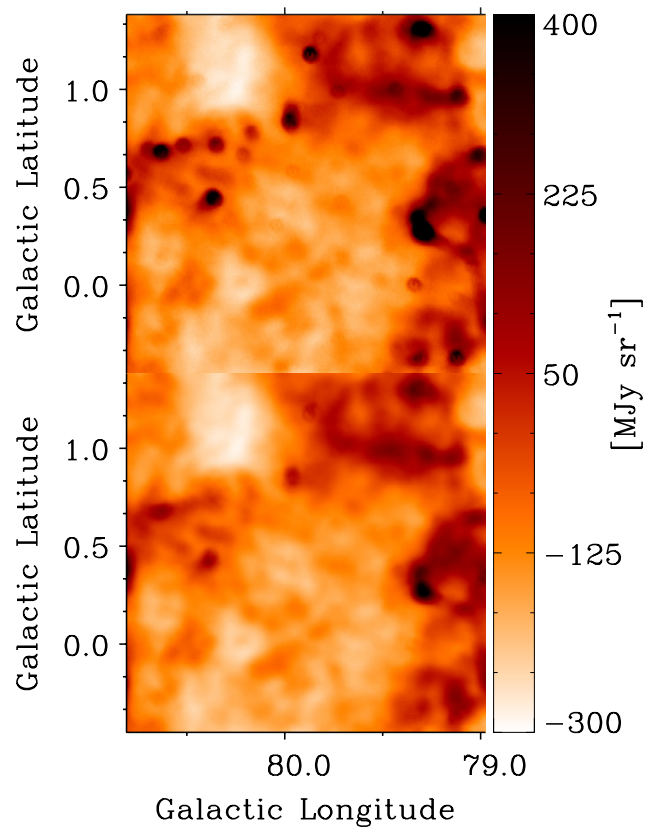

Figure 4. BLAST05 map at $250 \mu \mathrm{m}$ of the selected region of Cyg X used for studying diffuse emission. The map in the upper panel includes point sources which, due to the corrupted BLAST05 beam, are compact structures of size $\sim 3$ '3. In the lower panel, the sources have been removed.

(A color version of this figure is available in the online journal.)

and more easily identified. They are fairly well described by Gaussians. We fit Gaussians (multiple if crowded) to obtain flux densities, positions, and FWHM (major axes and position angle) of the compact sources. We then convolve these Gaussians with the synthetic beam and subtract them from the original maps. For the Cyg X sub-field analyzed, the upper and lower panels of Figure 4 show BLAST maps at $250 \mu \mathrm{m}$ before and after source removal, respectively. Faint residuals appear near some of the brightest sources, because of multiscale structure in the ISM in which they are embedded.

\subsection{Two-dimensional Power Spectrum}

Figure 5 shows the two-dimensional power spectrum for the Cyg X region at $250 \mu \mathrm{m}$. Because $\alpha \sim-3$ produces a large intrinsic dynamic range in the power spectrum, we have multiplied it by $\left(k / k_{0}\right)^{3}$, with $k_{0}=0.1 \operatorname{arcmin}^{-1}$. The dark rings are produced by the same features giving the dips in $\Gamma(k)$. Noise is being amplified at large $k$ because of the $k^{3}$ multiplier.

The two-dimensional power spectrum is circularly symmetric, justifying the annular averaging to find the mean $P(k)$. Most of the information relevant to the diffuse emission is contained in the plateau in the central region of Figure 5, and the average there in this representation is close to $P\left(k=0.1 \operatorname{arcmin}^{-1}\right)$.

The error in $P(k)$ for each annulus is the standard error of the mean. While removing the effect of beam from the power spectrum, we have only scaled this error by the inverse beam, not accounting for the fact that its shape is not perfectly known at high $k$. However, this is of no consequence because we do not fit the data at large $k$ where the beam correction is significant.

\subsection{Exponent and Amplitude}

Figure 6 shows $P(k)$ for $250 \mu \mathrm{m}$ for the Cyg X region, for the original map and with sources removed. The effect of the beam is dramatic at high $k$, showing that it is important to have

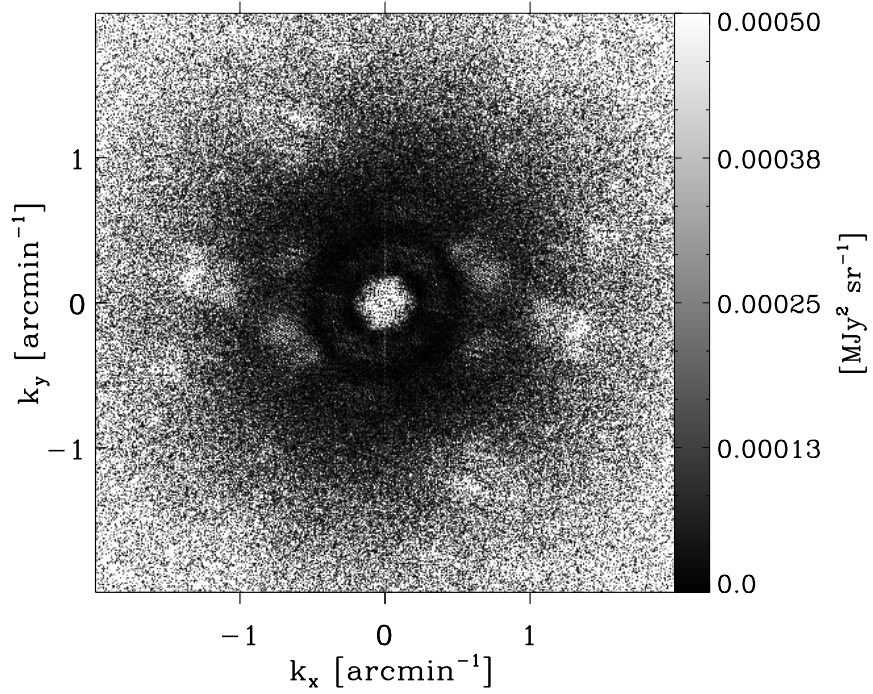

Figure 5. Two-dimensional power spectrum multiplied by $\left(k / k_{0}\right)^{3}$ (with $k_{0}=0.1 \operatorname{arcmin}^{-1}$ ), for the Cyg X map with compact sources removed, as shown in the lower panel of Figure 4. Dark rings are an imprint from the BLAST05 PSF (Figure 3). Scaling by $k^{3}$ has amplified noise at large $k$ and highlighted the bright central plateau, which contains the information most relevant for this study.

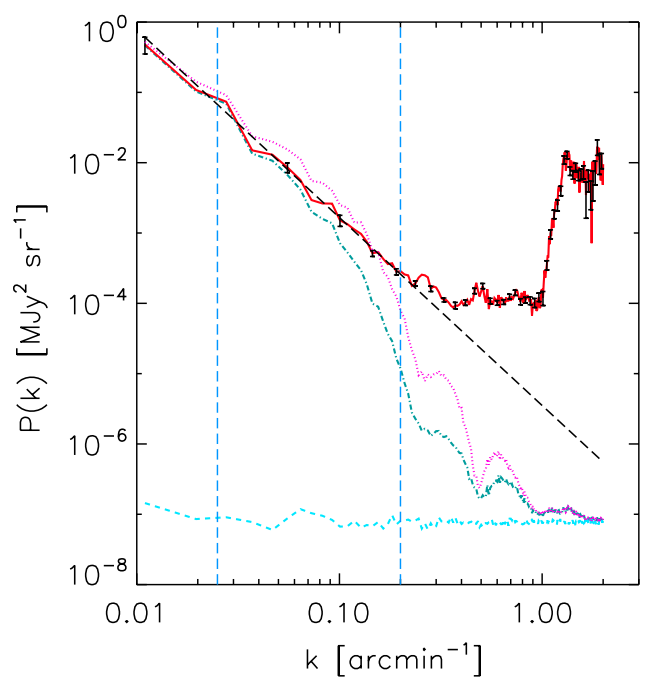

Figure 6. BLAST power spectra at $250 \mu \mathrm{m}$ for the Cyg $\mathrm{X}$ region shown in Figure 4. The dotted curve, corresponding to the upper panel of Figure 4, shows that a significant amount of power is present at intermediate scales due to compact sources. The lower dot-dash line is $P(k)$ of the same region after removing sources (corresponding to Figure 5). The oscillatory behavior at large $k$ is due to the corrupted BLAST05 PSF (Figure 3). The almost horizontal dashed line is the power spectrum of the noise map (Section 5.1). At very small scales where the astronomical signals become correlated within the beam, the power spectra meet the noise level. The solid line is the power spectrum $P_{\text {cirrus }}(k)$, after subtracting the noise and dividing by $\Gamma(k)$ to remove the effect of the beam (as in Figure 1, error bars at high $k$ do not include the imperfect knowledge of the beam and noise). The vertical dashed lines indicate the restricted range $0.025 \operatorname{arcmin}^{-1}<\mathrm{k}<0.2 \mathrm{arcmin}^{-1}$ used for fitting the power law. The exponent is $-2.60 \pm 0.07$ and $P\left(0.1 \mathrm{arcmin}^{-1}\right)=(1.60 \pm 0.05) \times$ $10^{-3} \mathrm{MJy}^{2} \mathrm{sr}^{-1}$.

(A color version of this figure is available in the online journal.)

a good estimate of the PSF. There is also clearly excess power due to sources and so it is important to remove them carefully.

At small $k$, the power spectrum is dominated by the dust structures; it decays toward higher $k$, as seen previously in IRIS data at shorter wavelengths. The oscillatory effect of $\Gamma(k)$ can be seen clearly at $k>0.2 \operatorname{arcmin}^{-1}$ and at higher $k$ the 


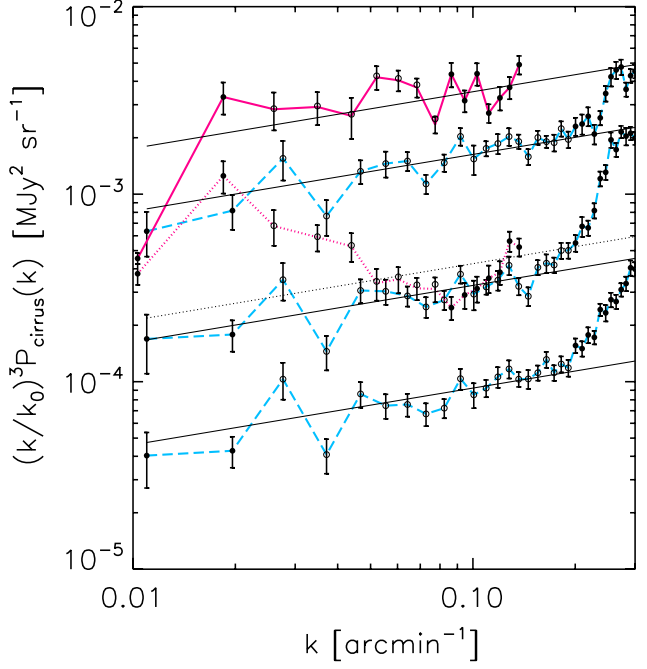

Figure 7. $\left(k / k_{0}\right)^{3} P_{\text {cirrus }}(k)$ for the three BLAST bands and two IRIS bands, for the Cyg X field with $k_{0}=0.1 \operatorname{arcmin}^{-1}$. Data at high $k$ (solid circles) are not used (or shown for the IRAS bands) because of the large and uncertain beam correction and eventually the dominance of noise (see Figures 2 and 6). The exponents are similar for all bands. The amplitude increases with decreasing wavelength for the BLAST bands and IRIS $100 \mu \mathrm{m}$ band (solid), and then decreases at $60 \mu \mathrm{m}$ (dotted). Lines represent a power law with common exponent -2.69 fit to the data plotted with open circles.

(A color version of this figure is available in the online journal.)

power spectrum meets the independently predicted noise level shown.

$P_{\text {cirrus }}(k)$, obtained by dividing the source-removed, noisesubtracted power spectrum by $\Gamma(k)$, is also shown in Figure 6 . It appears to have a power-law form over the limited dynamic range in $k$ available. We fit only over the range $0.025 \mathrm{arcmin}^{-1}<$ $\mathrm{k}<0.2 \operatorname{arcmin}^{-1}$, restricted for the reasons discussed in Section 4. In particular, at large $k$ the effect of correcting for the PSF is very large. The synthetic PSF is an average for the entire observed field and so is imperfectly modeled to the precision that would be required for precise compensation at high $k$. Convincing evidence for any deviation from a power law for the higher $k$ range will have to await the higher resolution observations anticipated with Herschel which will probe to beyond $k=1$. At smaller $k$, the observed power spectrum is affected both by apodization and by the effective filtering of the map-making procedure, such that the largest scales are not recovered. For the relatively small size of these maps and the characteristics of these BLAST05 observations, these effects set in at about the same $k$ (Patanchon et al. 2008). In earlier work with IRAS, the amplitude $P_{0}$ is often cited for $k_{0}=0.01 \mathrm{arcmin}^{-1}$. This fiducial value is below the range of our observations. Using $k_{0}=0.1 \mathrm{arcmin}^{-1}$ would seem preferable and probably more relevant to small maps at higher resolution to be made with Herschel.

Figure 7 shows $\left(k / k_{0}\right)^{3} P_{\text {cirrus }}(k)$ for all three BLAST bands $(250,350$, and $500 \mu \mathrm{m})$ for Cyg X. We find $P\left(0.1 \mathrm{arcmin}^{-1}\right)=$ $(1.60 \pm 0.05) \times 10^{-3} \mathrm{MJy}^{2} \mathrm{sr}^{-1}$ at $250 \mu \mathrm{m}$. $P_{0}$ increases with decreasing wavelength for the BLAST bands and for the IRIS bands (Section 4) remains about the same at $100 \mu \mathrm{m}$ and then decreases at $60 \mu \mathrm{m}$. We obtain an exponent $\alpha$ equal to $-2.60 \pm 0.07$ at $250 \mu \mathrm{m}$. This can be compared with the value found above at $100 \mu \mathrm{m},-2.97 \pm 0.23$. The exponents are quite similar for all bands, except for $60 \mu \mathrm{m}$ which is discussed further in Section 6.1. If the power spectrum were less steep moving into the submillimeter, this would provide

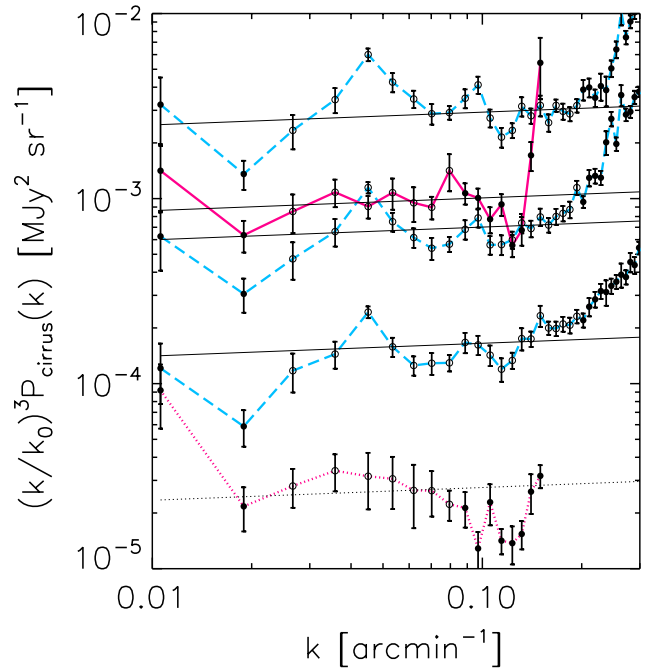

Figure 8. Same as Figure 7, but for the Aquila region. The amplitudes at 100 and $60 \mu \mathrm{m}$ are relatively lower, which indicates that the cirrus in this region is cooler. The common exponent used for the fits was -2.93 .

(A color version of this figure is available in the online journal.)

important evidence for a change in the statistical properties of the emission and the underlying mass column density and temperature distribution (Section 6.1). Definitive searches for such wavelength dependence should benefit from the higher spatial dynamic range anticipated in observations with Herschel.

Figure 8 presents the results from our analysis of the Aquila field. Overall the same behavior is seen, though the exponent appears to be slightly steeper $(-3.14 \pm 0.10)$ at $250 \mu \mathrm{m}, P_{0}$ at $250 \mu \mathrm{m}$ is larger, $(3.03 \pm 0.15) \times 10^{-3} \mathrm{MJy}^{2} \mathrm{sr}^{-1}$, and the relative amplitudes at the IRIS bands are much lower.

\subsection{Catalog Depth}

Equation (4) can be used to predict the depth reached in a compact source catalog. To apply this to the BLAST05 Cyg X survey (where the compact sources are of apparent size $\sim 1.5$ in the $\mathrm{L}-\mathrm{R}$ maps in which they are detected and measured), the appropriate $r$ is $\sim 3.6$. With $D=1.9 \mathrm{~m}$ and $P\left(0.1 \operatorname{arcmin}^{-1}\right)=$ $(1.60 \pm 0.05) \times 10^{-3} \mathrm{MJy}^{2} \mathrm{sr}^{-1}$ for the somewhat dimmer part of the survey field examined here, $\sigma_{\text {cirrus }}=4.2 \mathrm{Jy}$ at $250 \mu \mathrm{m}$. This compares well to the empirically estimated $3 \sigma$ detection threshold of $\sim 15 \mathrm{Jy}$ (A. Roy et al. 2010, in preparation). The effective noise near bright sources can be somewhat higher because of contaminating artifacts produced in the $\mathrm{L}-\mathrm{R}$ deconvolution.

For the large survey of Vela carried out by BLAST in 2006, where the beam of the $1.8 \mathrm{~m}$ mirror was diffraction limited (Netterfield et al. 2009), we have not measured $P_{0}$ directly, because the region was not covered by orthogonal scanning and so the cirrus structure is not well constrained over all scales in the cross-scan direction (Patanchon et al. 2008). Nevertheless, following the scaling suggested in Section 6, we can estimate $P_{0}$ from $\left\langle I_{100}\right\rangle \sim 100 \mathrm{MJy} \mathrm{sr}^{-1}$ and $T_{\mathrm{d}} \sim 18 \mathrm{~K}$, giving $P\left(0.1 \operatorname{arcmin}^{-1}\right)=10^{-3} \mathrm{MJy}^{2} \mathrm{sr}^{-1}$ at $250 \mu \mathrm{m}$, from which $\sigma_{\text {cirrus }} \sim 0.6 \mathrm{Jy}$. However, the sources were actually extended, with typical apparent sizes of $1^{\prime}$, so that $r \sim 2.4$, and then $\sigma_{\text {cirrus }} \sim 1.6 \mathrm{Jy}$. For comparison, the catalog depth judged from simulations of completeness was $6 \mathrm{Jy}$ (Netterfield et al. 2009). We conclude that the depth is dominated by the influence of the cirrus noise.

With Herschel and assuming the source sizes are compatible with $r=1.6, \sigma_{\text {cirrus }}$ in the Cyg $\mathrm{X}$ region should be closer 

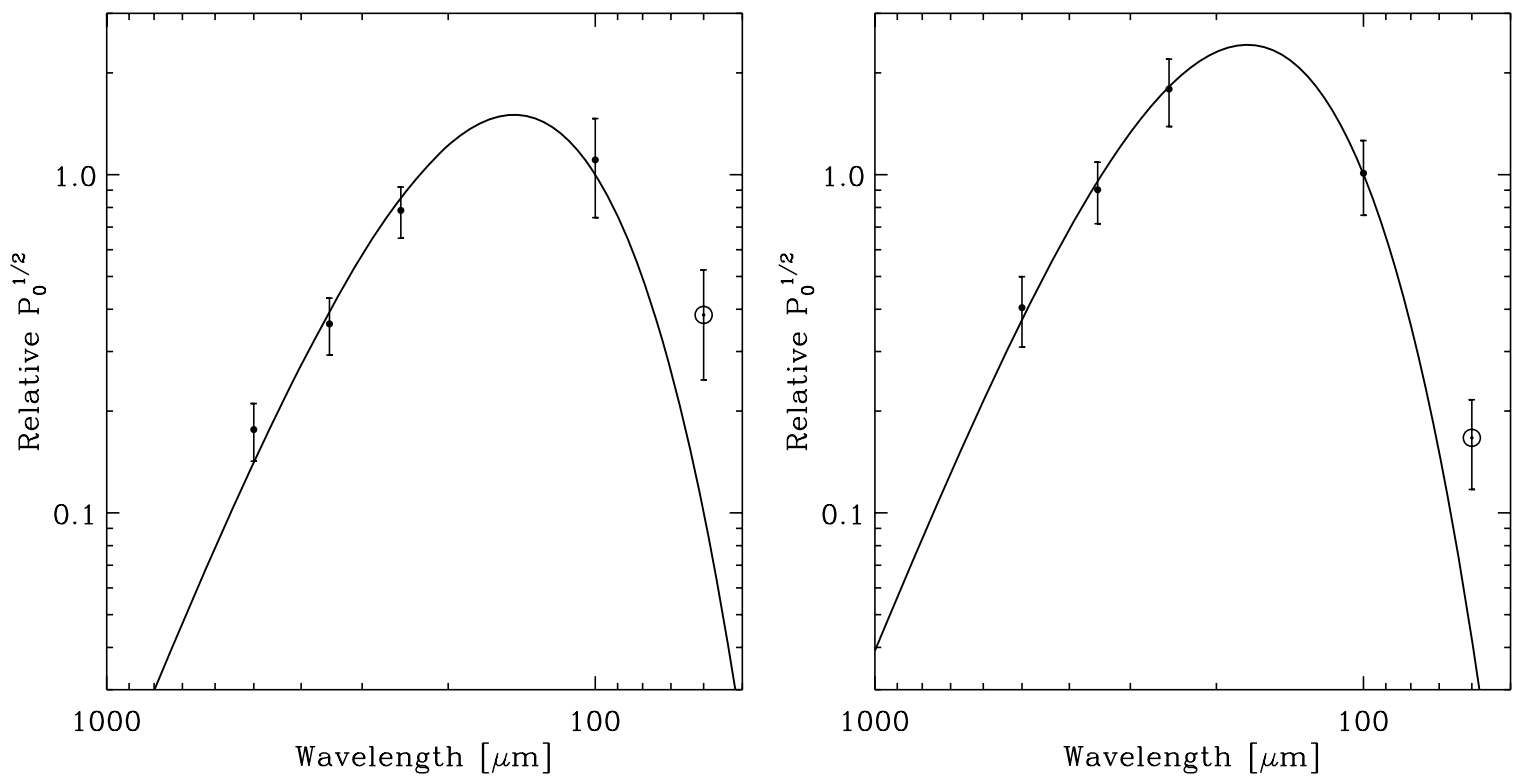

Figure 9. Relative SED obtained from the square root of the amplitudes of the power spectra fit in Figures 7 and 8. Left Cyg X; right, Aquila. The solid curve is the best-fit modified blackbody with $\beta=2$. The $60 \mu \mathrm{m}$ power spectrum values (open circles) were not used for the fits (see the text). The best-fit temperatures of Cyg X and Aquila are $19.8 \pm 1.5$ and $16.8 \pm 0.8 \mathrm{~K}$, respectively. For ease of comparing the two fields and results in Figures 10 and 11 , data and curves have been displayed normalized to the value of the best-fit modified blackbody at $100 \mu \mathrm{m}$.

to $100 \mathrm{mJy}$ at $250 \mu \mathrm{m}$. While a distinct improvement, this is nevertheless substantial compared to the instrument noise (rms $\sim 5 \mathrm{mJy}$ ) predicted using HSPOT $^{18}$ for the planned parallel PACS-SPIRE map scanning strategy in the guaranteed-time key project Herschel imaging survey of OB Young Stellar objects (HOBYS). ${ }^{19}$ We expect substantial regional variations. For example, in Aquila, simply from the larger $P_{0}$ the depth achieved should be 1.4 times worse. And this is not even the brightest part of the Galactic plane to be surveyed in the open-time key project Herschel infrared Galactic plane survey (Hi-GAL). ${ }^{20}$

Extraction of sources with wavelength-dependent and multiscale structure will be especially problematic and it will be necessary both to measure the cirrus properties and to simulate its effects. For SPIRE maps, in particular those made with the parallel PACS-SPIRE mode, a corollary is that the high signal-to-noise ratio resulting from the redundant coverage is well suited to studying the statistical properties of the bright cirrus. Of particular interest is how the power spectrum varies with wavelength, which can be used to determine physical properties of the diffuse dust.

\section{WAVELENGTH DEPENDENCE}

\subsection{Mass Column Density Distribution}

BLAST and IRAS maps record surface brightness $I$ which, in turn, depends on the dust mass column density $M_{\mathrm{d}}$ :

$$
I_{v}=M_{\mathrm{d}} \kappa_{v} B_{v}\left(T_{\mathrm{d}}\right)
$$

where $\kappa_{v}$ is the dust emissivity, $B_{v}$ is the Planck function for dust temperature $T_{\mathrm{d}}$ and it is understood that the right-hand side is summed over various dust components which might have different $\kappa$ and $T_{\mathrm{d}}$.

\footnotetext{
18 http://www.ipac.caltech.edu/Herschel/hspot.shtml

19 http://starformation-herschel.iap.fr/hobys/

20 https://hi-gal.ifsi-roma.inaf.it/higal
}

The underlying spatial property being probed is $M_{\mathrm{d}}$ which is in turn the projection of the three-dimensional density distribution. $M_{\mathrm{d}}$ is modulated as a function of frequency by the emissivity and temperature, and can also have modulations caused by spatial differences in these properties. The ISM in the Galactic plane is far from homogeneous, containing diffuse atomic and ionized regions and shielded molecular regions with possibly different mixes of dust compositions and sizes, and along any line of sight there will be a range of interstellar radiation field intensities, all of which could affect $T_{\mathrm{d}}$. The surface brightness $I_{v}$ is particularly sensitive to $T_{\mathrm{d}}$ for frequencies near or above the peak in the SED, i.e., for $100 \mu \mathrm{m}$ and shorter for typical interstellar diffuse dust temperatures. Therefore, it is somewhat surprising that the power spectra exponents that we obtained are so similar. The potential for wavelength dependence should be particularly high at $60 \mu \mathrm{m}$ where non-equilibrium emission from very small grains (VSGs) starts to become important (Desert et al. 1990; Li \& Draine 2001) and is even more directly responsive to the ultraviolet radiation field. The Cyg X field analyzed definitely has different spatial structures appearing in the images at the shorter wavelengths and there is some evidence in Figure 7 for an effect on the exponent of the power spectrum. Furthermore, the exponent for a particular region must depend, through the structure of $M_{\mathrm{d}}$, on the environments probed along the line of sight and the energy injection at large scales that is responsible for the apparent turbulence in the ISM.

In the simple case of homogeneous correlation in the ISM, $I_{v}$ everywhere in the map would scale with frequency according to the simple relative SED $S_{v}$ of the emitting dust. From Equation (5), $S_{v} \propto \kappa_{v} B_{v}\left(T_{\mathrm{d}}\right)$. Therefore, $P_{0}$, measuring the same structure in $M_{\mathrm{d}}$, would scale simply as $S_{v}^{2}$ (see Equation (1)) while $\sigma_{\text {cirrus }}$ would scale as $S_{v}$ (see Equation (4)). This scaling is what is assumed by the prescriptions of Lagache \& Puget (2000) and Miville-Deschênes et al. (2007), for example, and appreciated by Jeong et al. (2005). However, on making the substitution $P_{100} \propto\left\langle I_{100}\right\rangle^{3}$, Helou \& Beichman (1990) end up assuming that $P$ scales as $S^{3}$ and thus $\sigma_{\text {cirrus }}$ scales as $S^{1.5}$. 

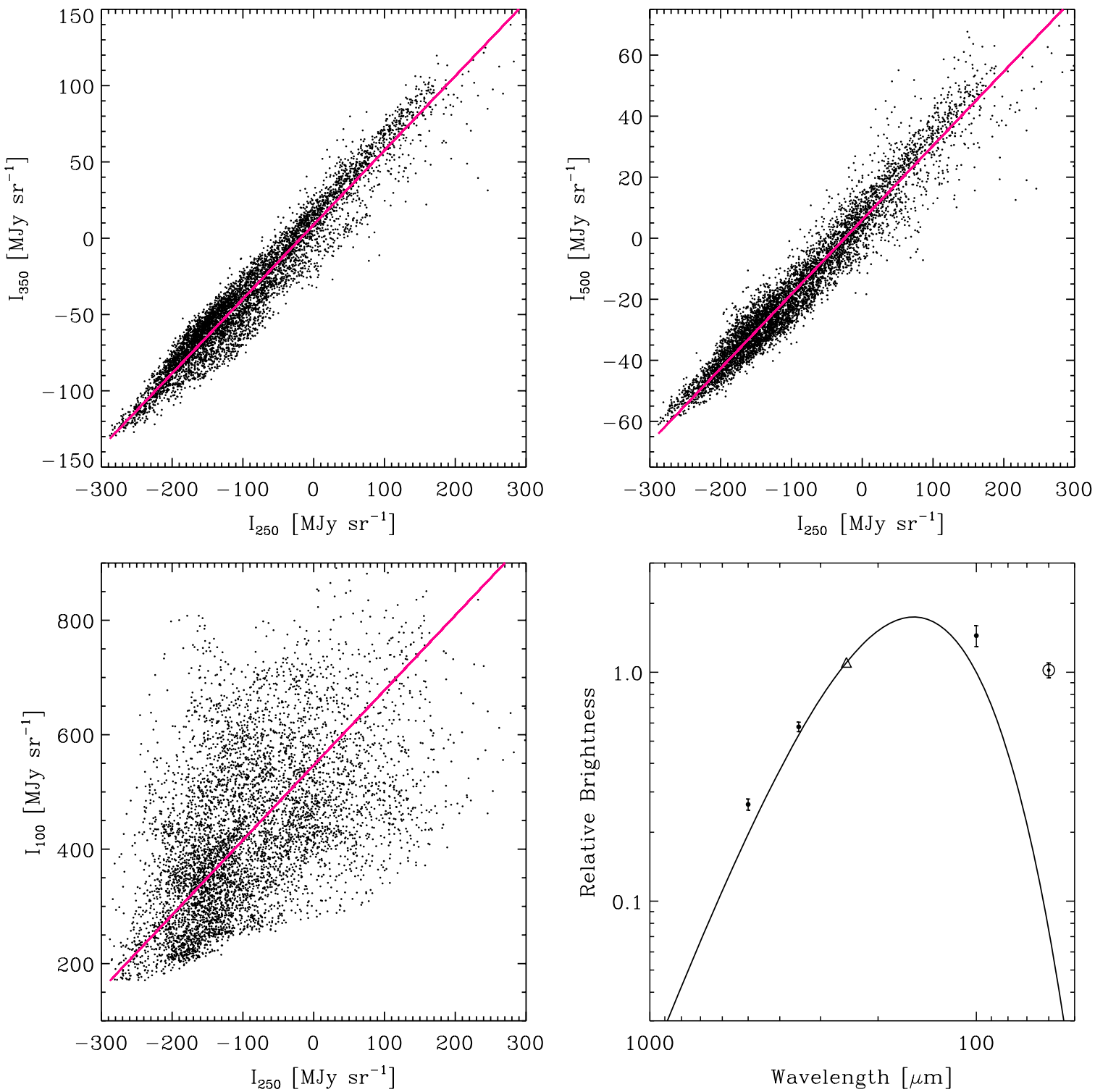

Figure 10. Pixel-pixel correlation of 350, 500, and $100 \mu \mathrm{m}$ maps with respect to the $250 \mu \mathrm{m}$ map for the Cyg X region, with the best-fit correlation line plotted. The lower right panel shows a relative SED obtained from the slopes of these correlations. With $\beta=2$, the best-fit temperature is $18.6 \pm 0.5 \mathrm{~K}$. The normalization here is the same as in Figure 9. The value at $250 \mu \mathrm{m}$, indicated by the triangle in this normalization, is not used explicitly for the fit. The open circle (from the $60 \mu \mathrm{m}$ slope) was also not used.

(A color version of this figure is available in the online journal.)

Kiss et al. (2001) found some empirical support for the latter over the ISO ISOPHOT wavelength range.

Note that the wavelength dependence of the signal-to-noise ratio of a point source will vary by the factor $(D / \lambda)^{2.5}$ from Equation (4), and also the ratio of the SED of the source relative to that of the confusing cirrus. At submillimeter wavelengths, the situation is more favorable for detecting cold sources in warm cirrus than vice versa. Therefore, the completeness depth of a survey as a function of wavelength will depend on these factors. Source size and structure can also change with wavelength (Netterfield et al. 2009) but we will not dwell on this quantitatively here.

\subsection{Characteristic Temperature}

The similarity in the exponents of the power spectra as a function of wavelength is no guarantee of underlying homo- geneous conditions. Nevertheless, we can assume that there is some characteristic SED for each region, and use the relative frequency dependence of $P_{0}^{1 / 2}$ to recover it. To be consistent with this picture, we evaluated $P\left(0.1 \operatorname{arcmin}^{-1}\right)$ using a common exponent, which is simply the average of the exponents found at each wavelength (excluding $60 \mu \mathrm{m}$ ). This relative SED is shown in Figure 9 for each region.

Fitting this relative SED with a modified black body (Equation (5)) yields a characteristic dust temperature. We have assumed $\kappa_{\nu} \propto v^{\beta}$ with $\beta=2$ (Hildebrand 1983). We did not include the $60 \mu \mathrm{m}$ data, because the excess there, presumed to be from non-equilibrium emission, would be inconsistent with a simple single-temperature modified black body. For Cyg X, the increase at long wavelengths with respect to the fit is probably the result of the range of temperatures known to be present in different parts of this region (A. Roy et al. 2010, in preparation). Therefore, even in this case we kept 

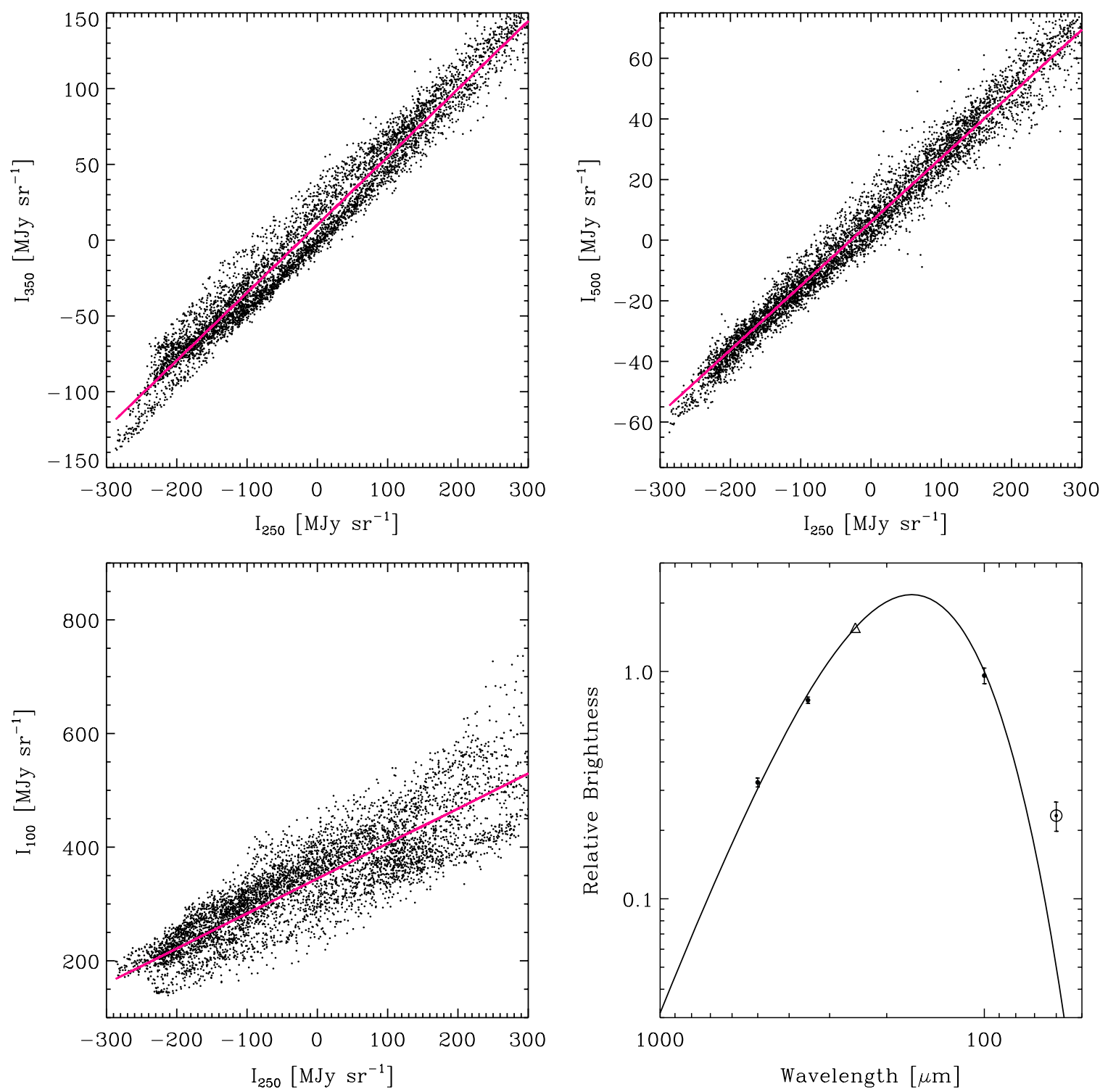

Figure 11. Same as Figure 10, but for the Aquila region. The slope of the $100 \mu \mathrm{m}$ correlation is much shallower than in the Cyg X field, and the best-fit temperature is $17.3 \pm 0.3 \mathrm{~K}$.

(A color version of this figure is available in the online journal.)

$\beta=2$, although empirically $\beta=1.5$ would produce a better fit.

For Cyg X, the best-fit temperature is $19.8 \pm 1.5 \mathrm{~K}$, which appears to be slightly warmer than the local high-latitude diffuse medium dust temperature of $17.5 \mathrm{~K}$ (Boulanger et al. 1996). Finding warmer dust in Cyg $\mathrm{X}$ is not particularly surprising, because this region, which lies along the local spiral feature $(l=$ $80^{\circ}$ ), is one of active star formation, with an $\mathrm{OB}$ association and numerous $\mathrm{H}$ II regions. In the $21 \mathrm{~cm}$ radio continuum, this region includes areas of strong diffuse thermal emission. To account for the higher equilibrium temperature, the ambient radiation field absorbed in Cyg X would have to be about $(19.9 / 17.5)^{6}=2.1$ times higher than locally.

By contrast, for Aquila the characteristic temperature derived is somewhat lower, about $16.8 \pm 0.8 \mathrm{~K}$. This line of sight passes through the inner Galaxy $\left(l=45^{\circ}\right)$, with significant molecular clouds but much less star formation (A. Rivera-Ingraham et al. 2010, in preparation).
As we have noted, under uniform conditions, maps at different wavelengths will have to be highly correlated in their spatial structures, and so they will have the same power spectrum scaled by $S^{2}$. But the fact that the relative SED derived from $P_{0}^{1 / 2}$ appears reasonable is no guarantee that the maps are simply scaled versions of one another (since phases could be different). However, this can be checked directly. Inspection of the BLAST maps shows that they are remarkably similar in all three bands. This is quantified through the correlations with respect to the $250 \mu \mathrm{m}$ map shown in Figure 10 for Cyg X. The linear fits shown are the ordinary least-squares bisector solution (Isobe et al. 1990) obtained with the IDL routine SIXLIN. Note that the correlation of BLAST $250 \mu \mathrm{m}$ with IRIS $100 \mu \mathrm{m}$ is not as high as it is between BLAST bands. This is expected, because of the above-mentioned greater sensitivity of $100 \mu \mathrm{m}$ emission to $T_{\mathrm{d}}$. Differences with respect to the submillimeter images become more apparent at $60 \mu \mathrm{m}$, where in addition the power spectrum is found to be somewhat steeper (Figure 7). Figure 11 
shows the even better correlations for the Aquila region. A clue to understanding the good correlation is, of course, the very detailed similarity of the power spectra at the different wavelengths.

As a consistency check, the slopes of the correlations can be used to construct another relative SED. As seen in Figures 10 and 11, these look very similar to the respective SEDs based on the power spectra (Figure 9) and the best-fit temperatures $18.6 \pm 0.5 \mathrm{~K}$ and $17.3 \pm 0.3 \mathrm{~K}$ are very similar to those found above.

We therefore have some evidence that the appropriate scaling of $\sigma_{\text {cirrus }}$ is as $S$, not $S^{1.5}$. However, this is not immediately generally useful, unless there is some independent evidence about the frequency dependence of $S$ (or the effective $T_{\mathrm{d}}$ and $\beta$ ), since this can clearly vary significantly from region to region. The best approach is to measure $P_{0}$ directly at each wavelength.

\section{CONCLUSION}

In these fields in the Galactic plane, the exponent of the power spectrum of the $100 \mu \mathrm{m}$ IRIS map is close to -3 , within the dispersion seen by Miville-Deschênes et al. (2007) despite the average brightness $\left\langle I_{100}\right\rangle$ being beyond the range studied by these authors. On the other hand, the amplitudes of the $100 \mu \mathrm{m}$ power spectra estimated for these bright $\sim 2^{\circ} \times 2^{\circ}$ fields are significantly below what would be extrapolated from the trend with $\left\langle I_{100}\right\rangle$ found by Miville-Deschênes et al. (2007). Therefore, particularly in such bright star-forming regions, it is recommended that the power spectrum be computed directly. The power spectra derived from the BLAST observations are also well fit by power laws, with similar exponents. The frequency dependence of the amplitude of the power spectrum can be described by the square of an SED which is a simple modified black body function with a reasonable characteristic temperature. This is confirmed by direct correlations between the maps at different wavelengths. However, this characteristic temperature does appear to change in different Galactic environments, and unless its value is known independently the power spectra and/or map correlations need to be evaluated for all wavelengths.

Cirrus noise will be important in many planned multiwavelength Galactic plane, high-latitude, and extragalactic surveys carried out with Herschel. Our results provide important empirical support for a proposed prescription for the wavelength dependence of the cirrus noise, which incorporates a factor which varies directly as the SED. In practice, the noise will be best evaluated, most free of assumptions, simply by measuring $P_{0}^{1 / 2}$ at each wavelength and using Equation (4).

The BLAST collaboration acknowledges the support of NASA through grant numbers NAG5-12785, NAG5-13301, and NNGO-6GI11G, the Canadian Space Agency (CSA), the UK Particle Physics \& Astronomy Research Council (PPARC), and Canada's Natural Sciences and Engineering Research Council (NSERC). We thank the Columbia Scientific Balloon Facility (CSBF) staff for their outstanding work.

\section{REFERENCES}

Boulanger, F., Abergel, A., Bernard, J.-P., Burton, W. B., Desert, F.-X., Hartmann, D., Lagache, G., \& Puget, J.-L. 1996, A\&A, 312, 256

Chapin, E. L., et al. 2008, ApJ, 681, 428

Desert, F.-X., Boulanger, F., \& Puget, J. L. 1990, A\&A, 237, 215

Gautier, T. N., III, Boulanger, F., Perault, M., \& Puget, J. L. 1992, AJ, 103, 1313

Helou, G., \& Beichman, C. A. 1990, in Liege International Astrophysical Colloquia, Vol. 29, Liege International Astrophysical Colloquia, ed. B. Kaldeich, 117

Hildebrand, R. H. 1983, Q. J. R. Astron. Soc., 24, 267

Isobe, T., Feigelson, E. D., Akritas, M. G., \& Babu, G. J. 1990, ApJ, 364, 104

Jeong, W.-S., Mok Lee, H., Pak, S., Nakagawa, T., Minn Kwon, S., Pearson, C. P., \& White, G. J. 2005, MNRAS, 357, 535

Kiss, C., Ábrahám, P., Klaas, U., Juvela, M., \& Lemke, D. 2001, A\&A, 379, 1161

Kiss, C., Ábrahám, P., Klaas, U., Lemke, D., Héraudeau, P., del Burgo, C., \& Herbstmeier, U. 2003, A\&A, 399, 177

Lagache, G., \& Puget, J. L. 2000, A\&A, 355, 17

Li, A., \& Draine, B. T. 2001, ApJ, 554, 778

Markwardt, C. B. 2009, in ASP Conf. Ser. 411, Astronomical Data Analysis Software and Systems XVIII, ed. D. A. Bohlender, D. Durand, \& P. Dowler (San Francisco, CA: ASP), 251

Miville-Deschênes, M.-A., \& Lagache, G. 2005, ApJS, 157, 302

Miville-Deschênes, M.-A., Lagache, G., Boulanger, F., \& Puget, J.-L. 2007, A\&A, 469, 595

Miville-Deschênes, M.-A., Lagache, G., \& Puget, J.-L. 2002, A\&A, 393, 749

Netterfield, C. B., et al. 2009, ApJ, 707, 1824

Pascale, E., et al. 2008, ApJ, 681, 400

Patanchon, G., et al. 2008, ApJ, 681, 708

Schneider, N., Bontemps, S., Simon, R., Jakob, H., Motte, F., Miller, M., Kramer, C., \& Stutzki, J. 2006, A\&A, 458, 855

Truch, M. D. P., et al. 2008, ApJ, 681, 415

Truch, M. D. P., et al. 2009, ApJ, 707, 1723

Waller, W. H., \& Boulanger, F. 1994, in ASP Conf. Ser. 58, The First Symposium on the Infrared Cirrus and Diffuse Interstellar Clouds, ed. R. M. Cutri \& W. B. Latter (San Francisco, CA: ASP), 129 